\title{
Electronic and transport properties of topological material GdxSb2-xTe3
}

Firoza Kabir, Xiaxin Ding, M. MOfazzel Hosen, Narayan Poudel, Gyanendra Dhakal, Arjun Pathak, Madhab Neupane, Krzysztof Gofryk

July 2019

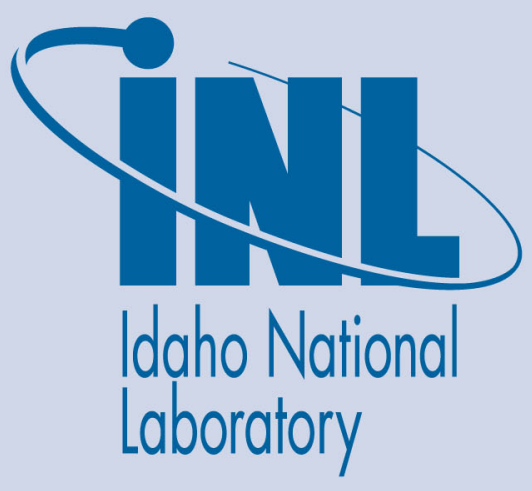

The INL is a U.S. Department of Energy National Laboratory operated by Battelle Energy Alliance 


\title{
Electronic and transport properties of topological material GdxSb2-xTe3
}

\author{
Firoza Kabir, Xiaxin Ding, M. MOfazzel Hosen, Narayan Poudel, Gyanendra \\ Dhakal, Arjun Pathak, Madhab Neupane, Krzysztof Gofryk
}

July 2019

Idaho National Laboratory Idaho Falls, Idaho 83415

http://www.inl.gov

Prepared for the U.S. Department of Energy Office of Nuclear Energy Under DOE Idaho Operations Office

Contract DE-AC07-05ID14517 


\section{Electronic and transport properties of topological material $\mathrm{Gd}_{\mathrm{x}} \mathrm{Sb}_{2-\mathrm{x}} \mathrm{Te}_{3}$}

Firoza Kabir ${ }^{1,2}$, Xiaxin Ding ${ }^{1}$, M. Mofazzel Hosen ${ }^{2}$, Narayan Poudel ${ }^{1}$,Gyanendra Dhakal ${ }^{2}$, Arjun Pathak ${ }^{3}$ Madhab Neupane ${ }^{2}$, Krzysztof Gofryk ${ }^{1}$

${ }^{1}$ Idaho National Laboratory, ${ }^{2}$ University of Central Florida, ${ }^{3}$ AMES laboratory

\section{Motivation}

Topological Insulator (TI): * Bulk band gap like an ordinary insulator but have protected conducting states on their edge or surface.

$>$ Due to spin-momentum locking, spin polarized electrons can move through surface.

$>$ Band inversion as a result of strong spin orbit coupling.
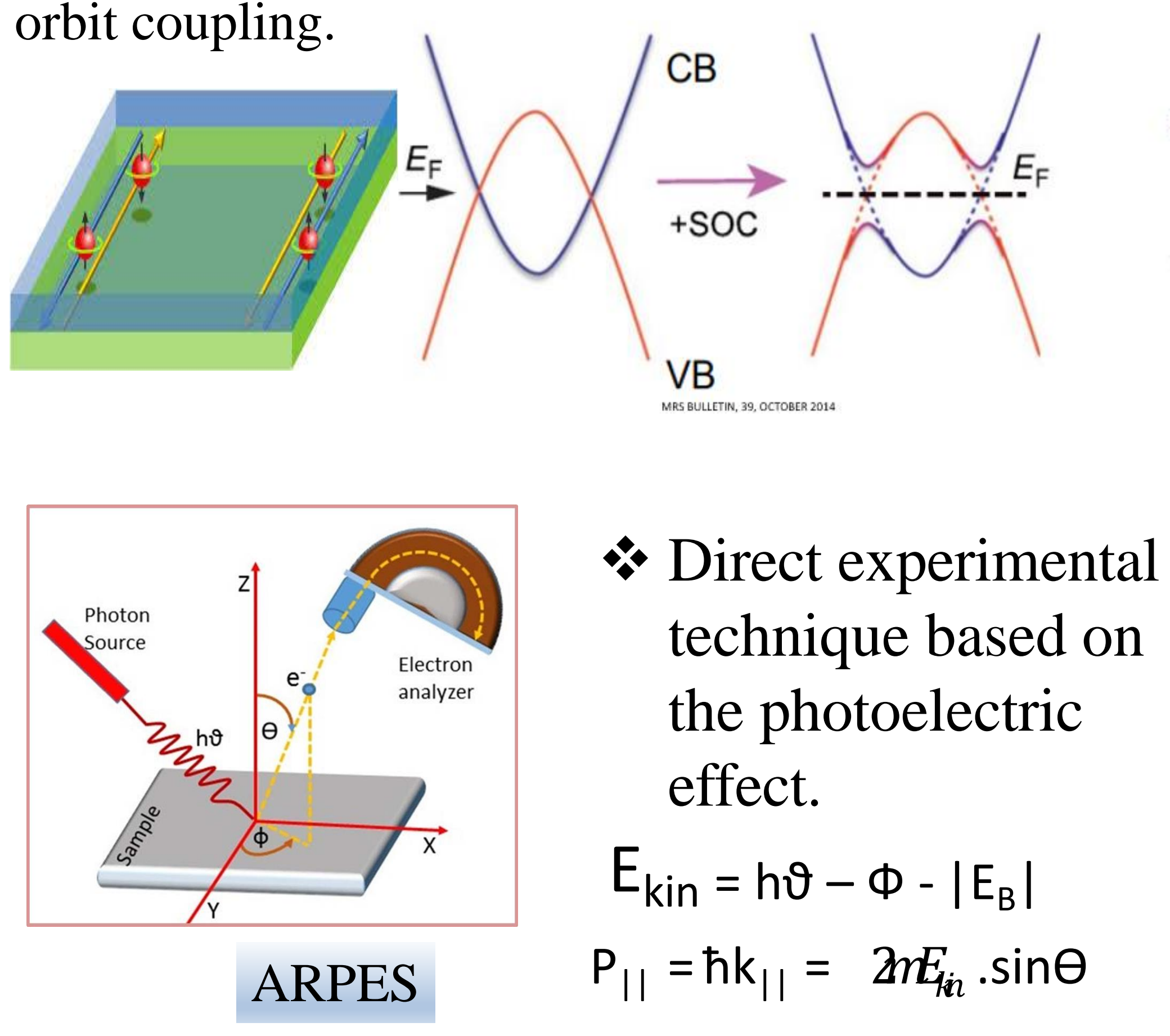

Gd doped TI $\left(\mathrm{Gd}_{\mathrm{x}} \mathrm{Sb}_{2-\mathrm{x}} \mathrm{Te}_{3}\right)$

* Trigonal, space group: R3m [166]

- Parent $\mathrm{Sb}_{2} \mathrm{Te}_{3}$ is a strong TI.

* Energy band gap 0.3 eV

- Crystal synthesis by flux method.

* Characterized by XRD and EDS.
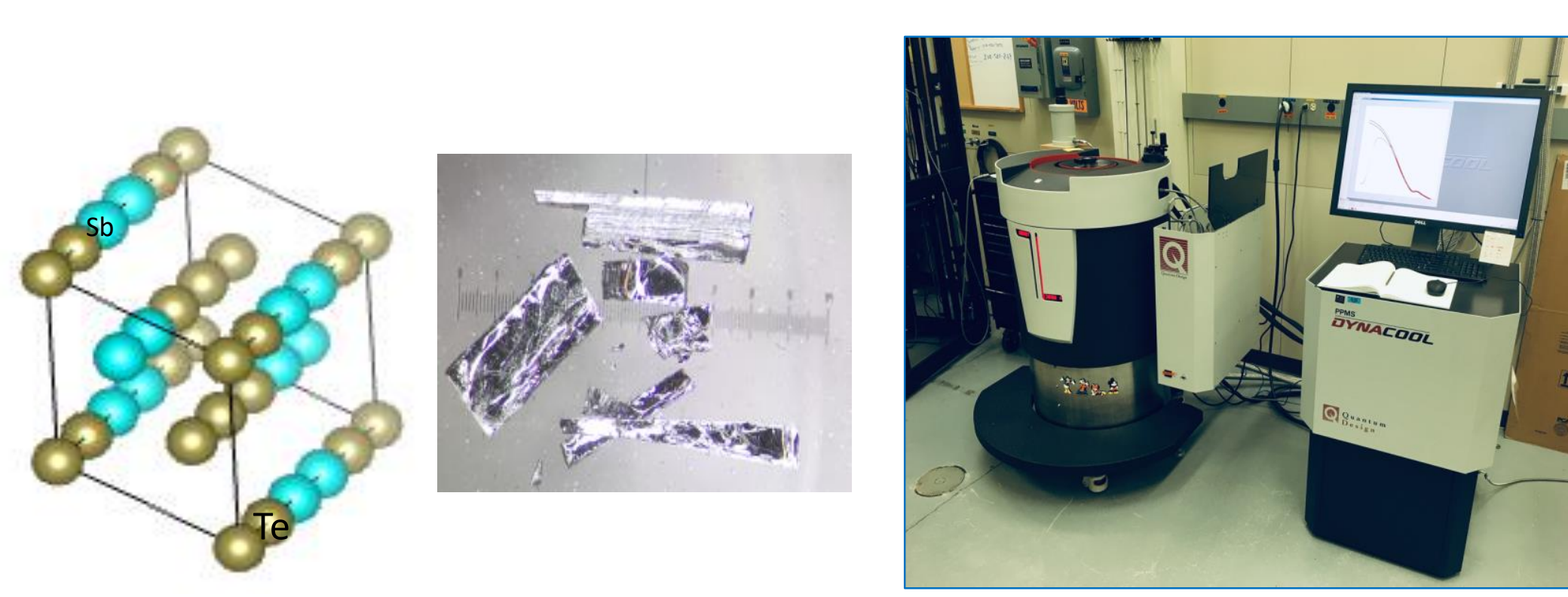

PPMS

$\checkmark$ Tuning Fermi energy by doping.

$\checkmark$ Studies of electronic properties of this material.

$\checkmark$ One of the best thermoelectric material.

$\checkmark$ Archetypical $\mathrm{TI}\left(\mathrm{Sb}_{2} \mathrm{Te}_{3}\right)$

Fermi surface and Dispersion map of $\mathrm{Gd}_{\mathrm{x}} \mathrm{Sb}_{2-\mathrm{x}} \mathrm{Te}_{3}$

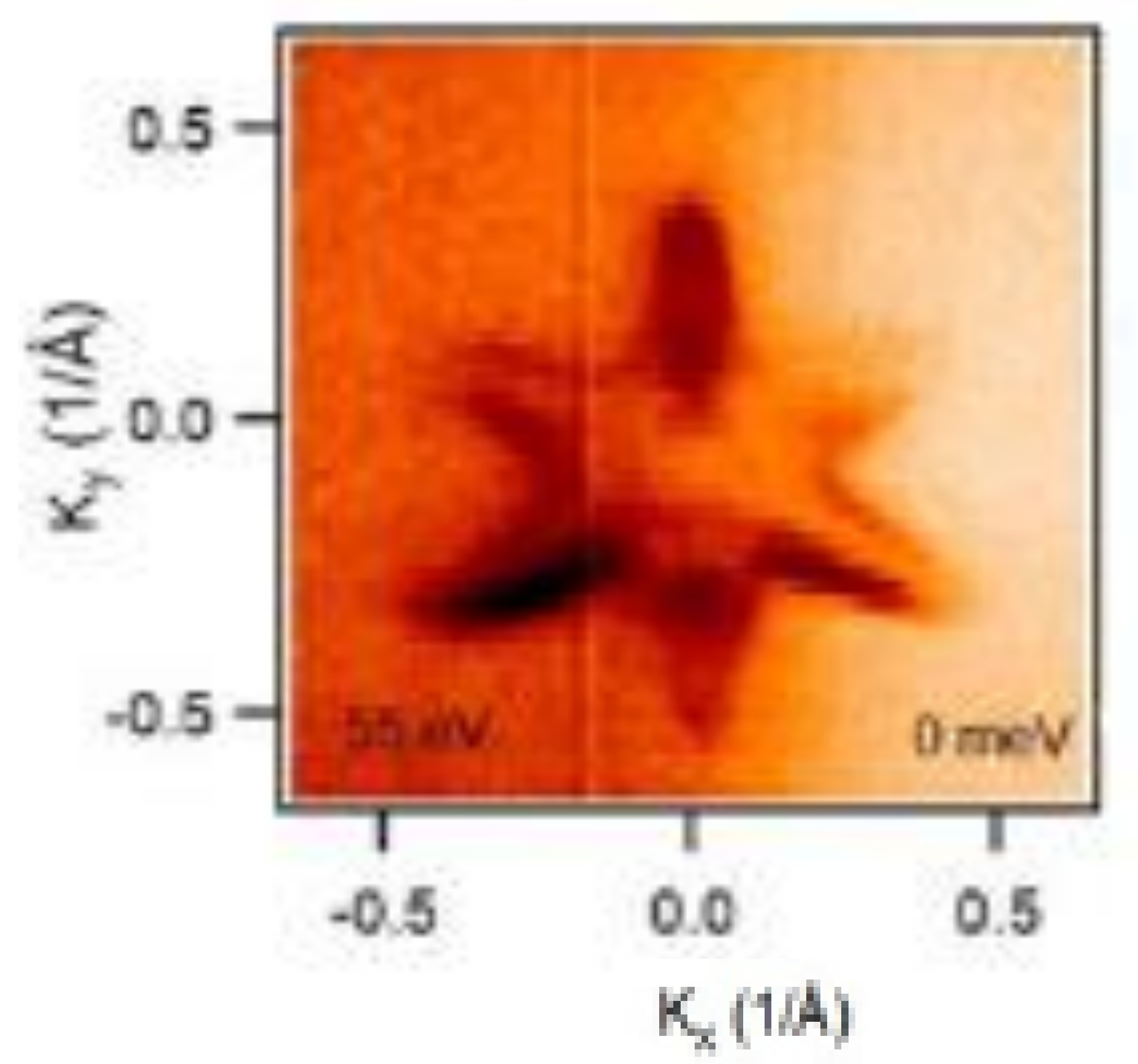

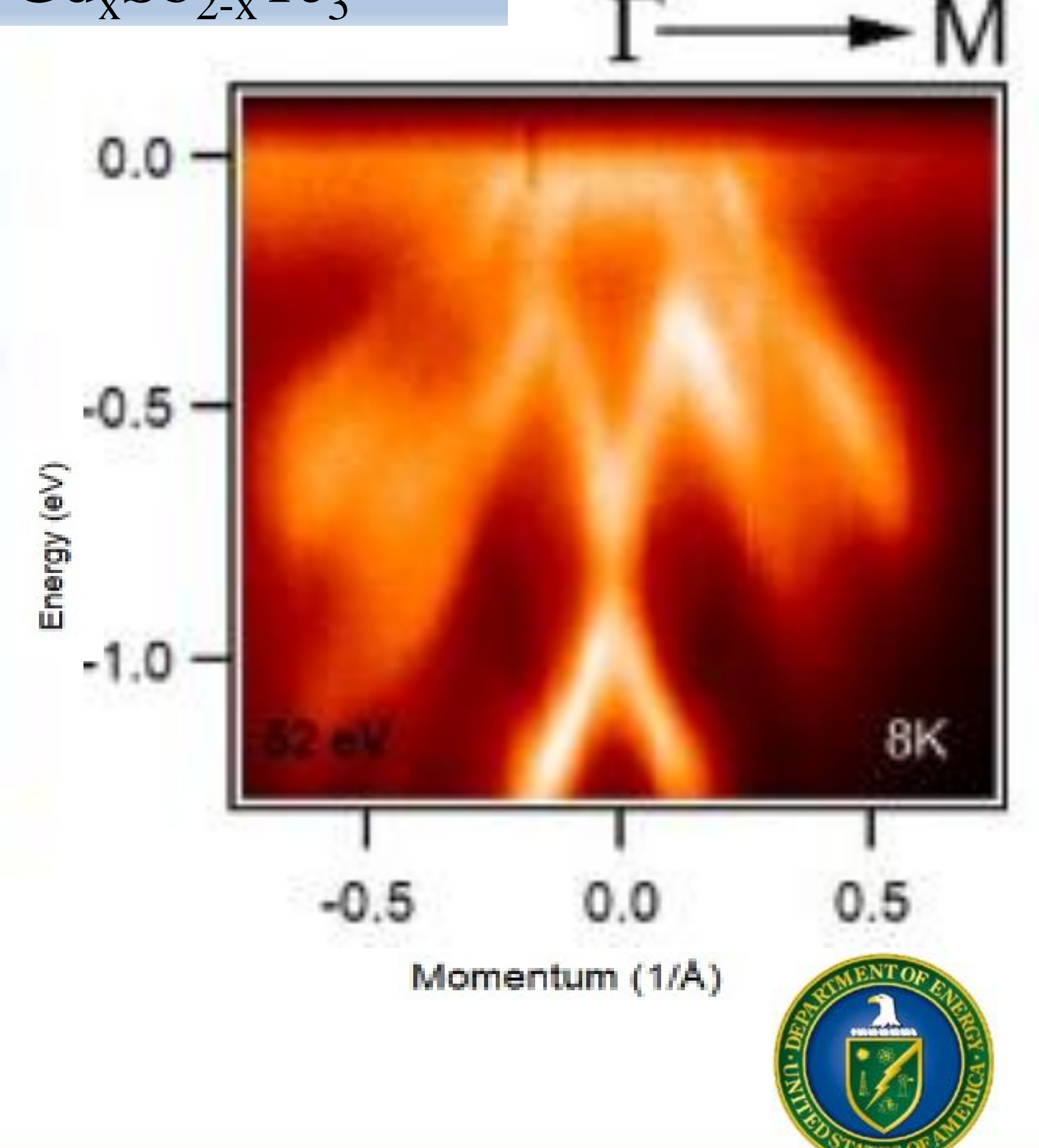

Transport and Magnetization properties of $\mathrm{Gd}_{\mathrm{x}} \mathrm{Sb}_{2-\mathrm{x}} \mathrm{Te}_{3}$ (a)

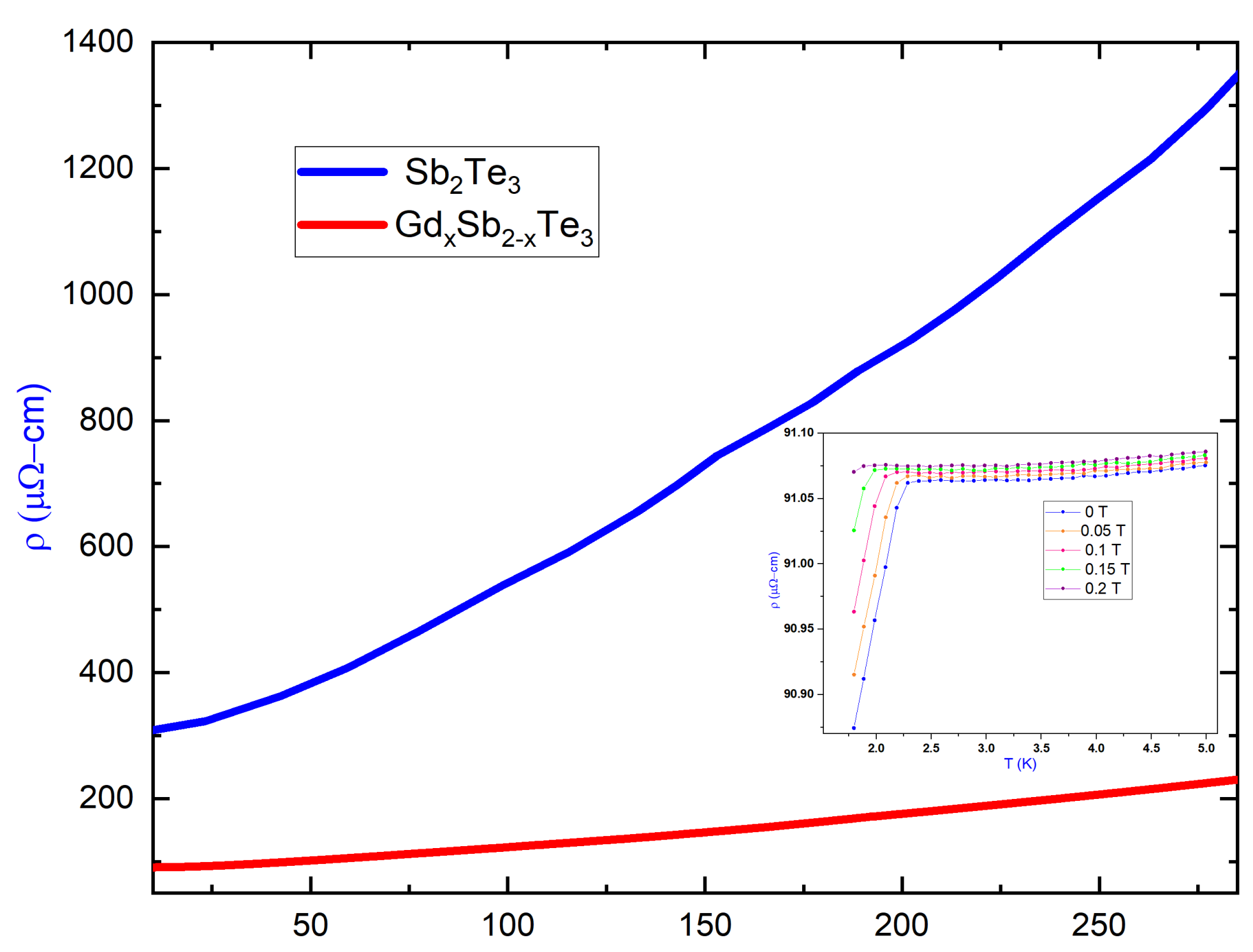

(c)

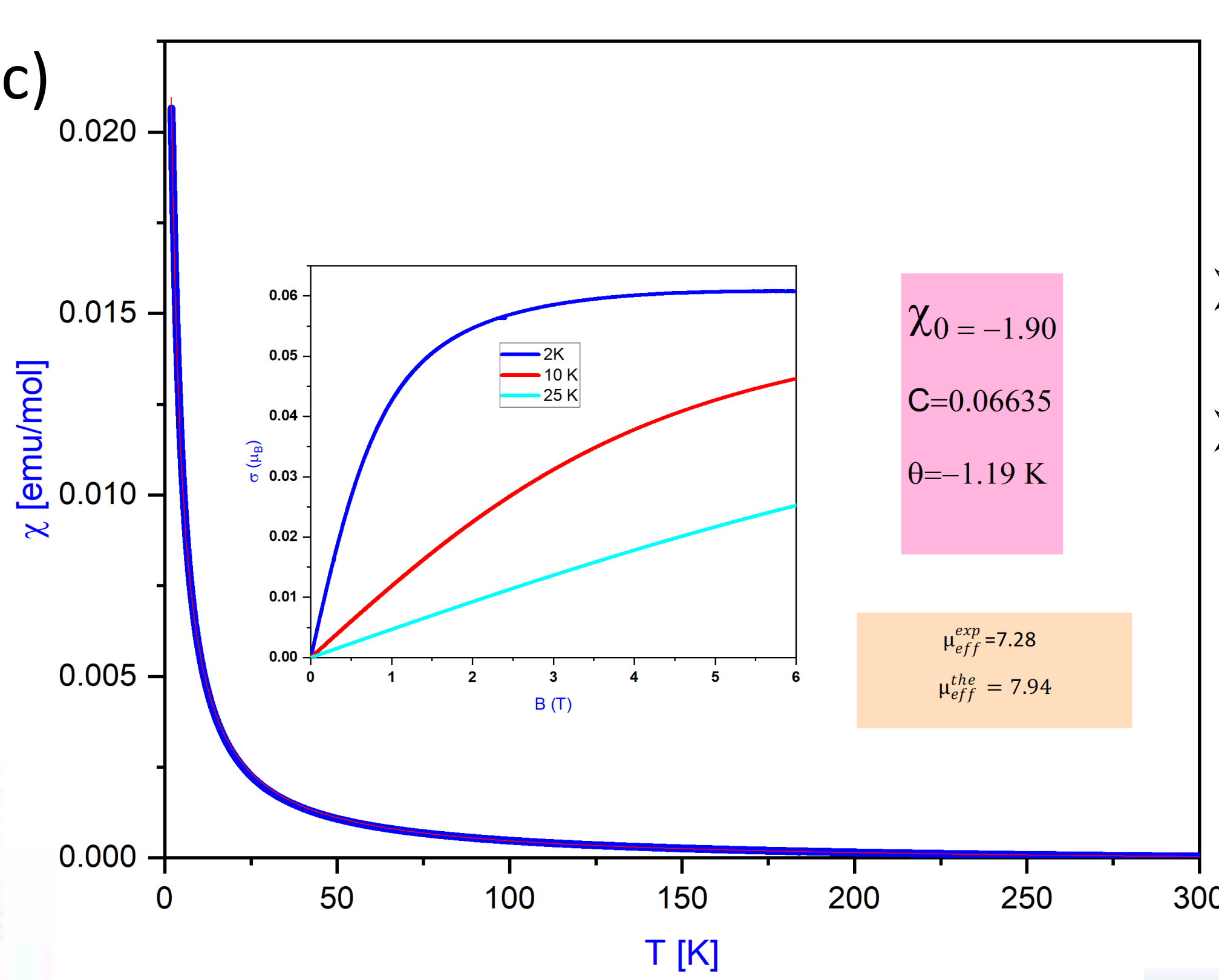

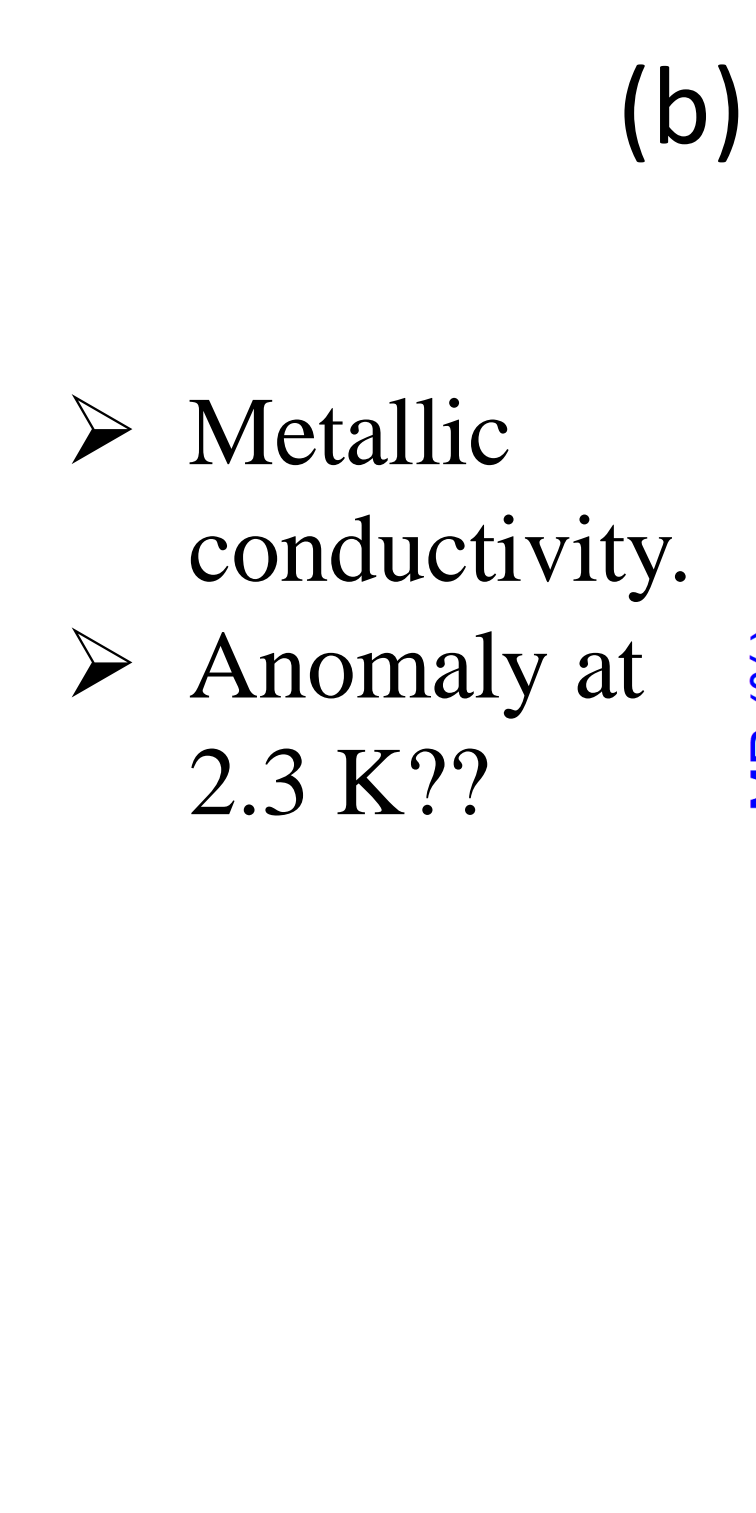

(b)

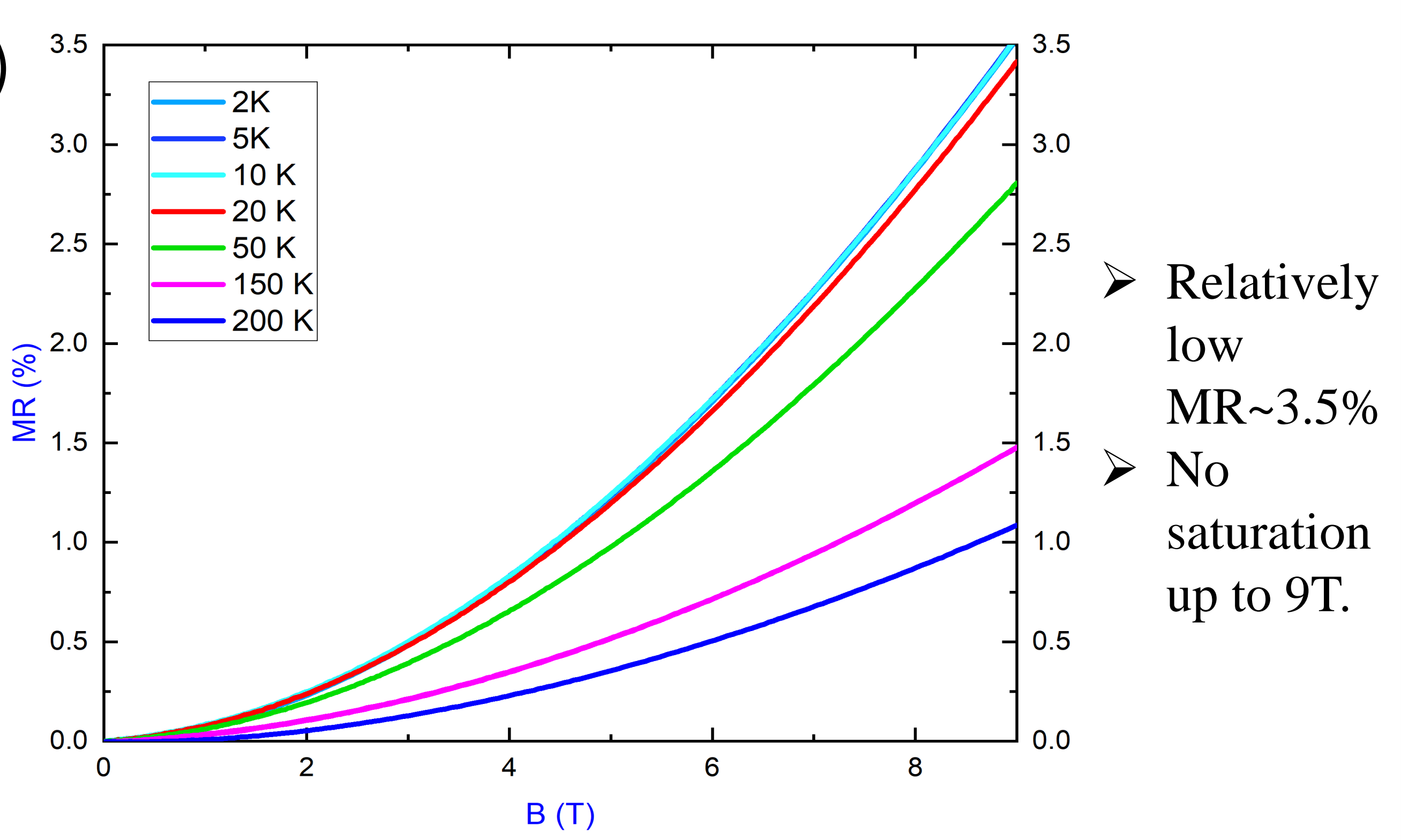

Hall resistivity and carrier concentration
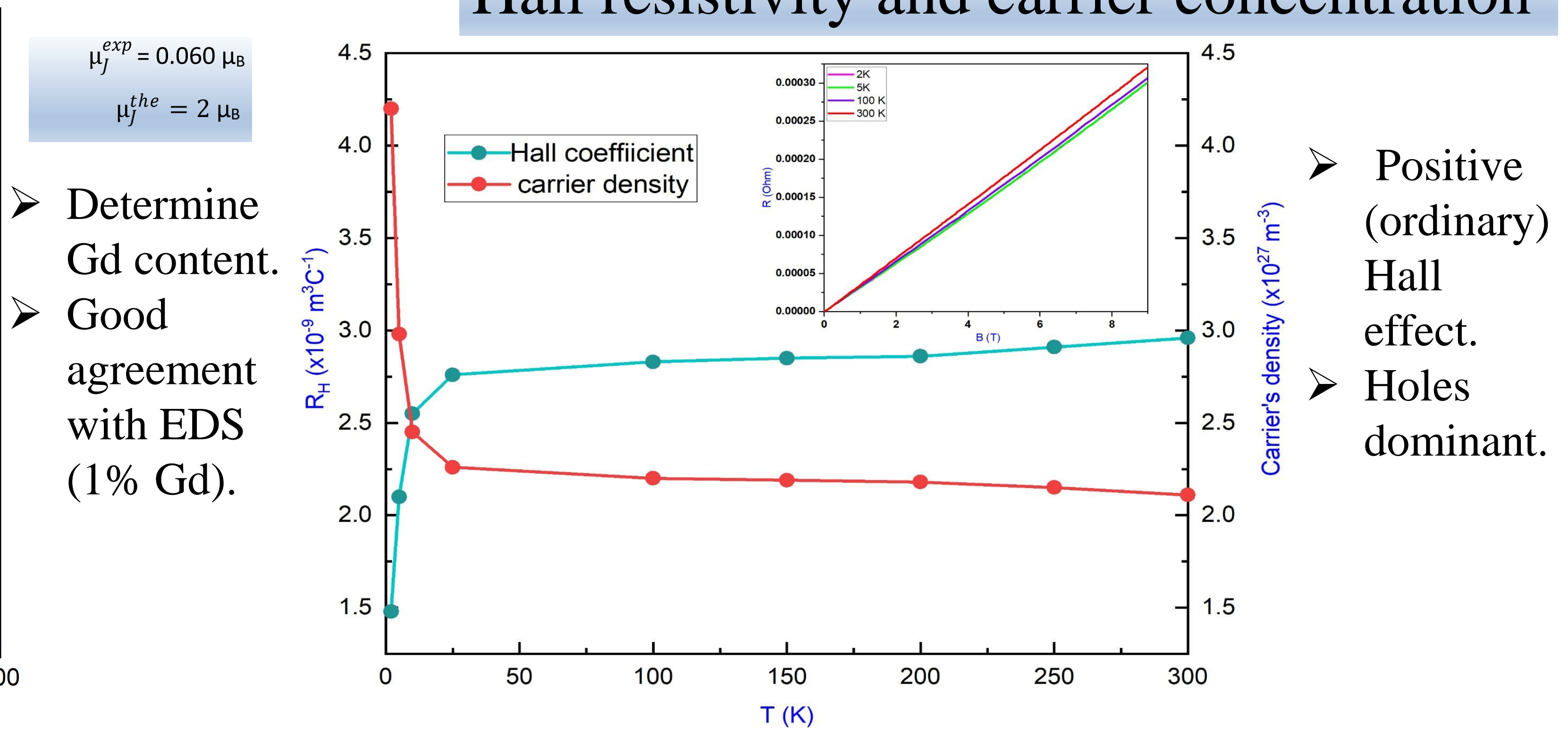

Heat capacity measurement

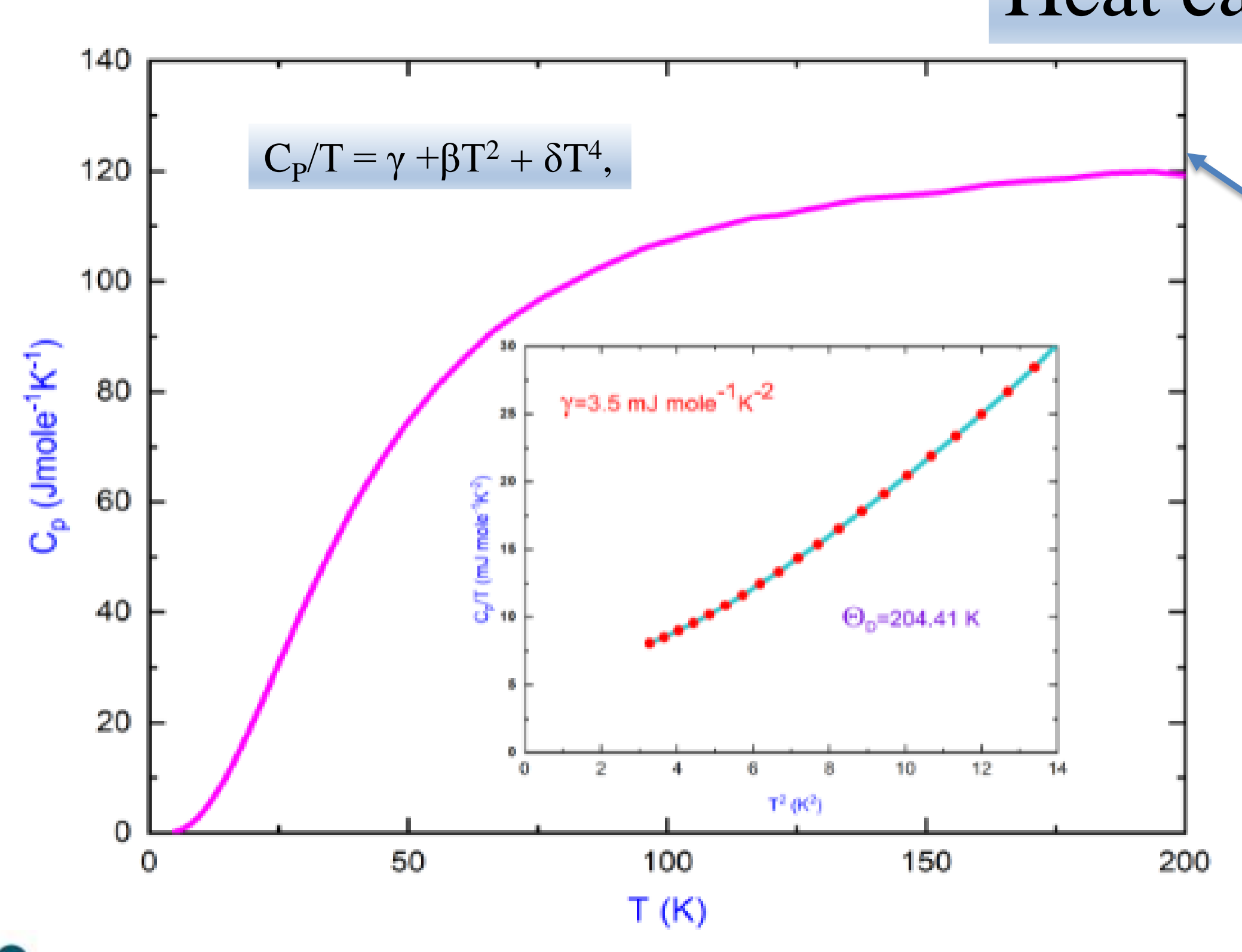

Dulong Petit limit

\section{Conclusion:}

- Successfully synthesized $\mathrm{Gd}_{\mathrm{x}} \mathrm{Sb}_{2-\mathrm{x}} \mathrm{Te}_{3}$

For parent

$\mathrm{Sb}_{2} \mathrm{Te} 3$,

$\gamma=0.7 \pm 0.7 \mathrm{~mJ}$

mole $^{-1} \mathrm{~K}^{-2}$

* Significant influence of Gd on electronic properties.

* Magnetism: excellent probe of small Gd concentration in $\mathrm{Gd}_{\mathrm{x}} \mathrm{Sb}_{2-\mathrm{x}} \mathrm{Te}_{3}$.

To be continued.... 